\title{
Selection of Hyper Exopolysaccharide Producing and Cyst Forming Azotobacter Isolates for Better Survival under Stress Conditions
}

\author{
S.L. Sivapriya and Pasupuleti Reddy Priya* \\ Department of Agricultural Microbiology, Tamil Nadu Agricultural University, \\ Coimbatore 641003, Tamil Nadu, India \\ *Corresponding author
}

\section{A B S T R A C T}

The present study was focused to select hyper exopolysaccharide producing and cyst forming Azotobacter isolates of rice from different locations of Tamil Nadu for better performance under the stress conditions prevailing in the rice ecosystem. The factors influencing cyst formation and exopolysaccharide (EPS) production in Azotobacter isolates and the performance of the isolates at different moisture regimes and fertilizer Nitrogen $(\mathrm{N})$ levels in rice under pot culture conditions were also investigated. Based on morphological and biochemical characterization, 10 efficient Azotobacter isolates were selected for evaluating exopolysaccharide production in comparison with two standard strains. Based on

Keywords

Exopolysaccharide, Azotobacter,

Survival and Stress Condition.

Article Info

Accepted:

26 May 2017

Available Online:

10 June 2017 exopolysaccharide production, three efficient Azotobacter isolates viz., AztRMD2 (1.755 $\mathrm{g} / 50 \mathrm{ml})$, AztPMK1 $(1.56 \mathrm{~g} / 50 \mathrm{ml})$ and AztPMK2 $(1.353 \mathrm{~g} / 50 \mathrm{ml})$ were selected for studying the cyst formation. The effect of limiting conditions of specific single micronutrient, combination of two micronutrients, reduced sucrose concentrations and addition of $\mathrm{n}$ - butanol as a carbon source on the induction of cyst formation was evaluated. The AztRMD2 showed maximum cyst formation of around 50 - 85 numbers in the growth medium without magnesium and also even when combinations of two micronutrients were eliminated from the growth medium, AztRMD2 formed 90 cysts $\mathrm{ml}^{-1}$. The salinity tolerance of AztRMD2 was evaluated by growing it in the growth medium containing different $\mathrm{NaCl}$ concentration. The results indicated that AztRMD2 can withstand upto $4 \% \mathrm{NaCl}$ concentration whereas maximum polysaccharide production was noticed in the broth containing $0.02 \%$ of $\mathrm{NaCl}$ concentration. For artificial soil aggregation, AztRMD2 showed maximum pore space percentage $(52.5 \%)$ and minimum bulk density $(1.12 \mathrm{~g} / \mathrm{cc})$. The aggregation stability (45stable aggregates) was also high in this isolate compared to Control. Hence based on these desirable characters such as exopolysaccharide production, cyst formation, salinity tolerance and soil aggregation, AztRMD2 was selected for studying its performance under different moisture regimes and fertilizer $\mathrm{N}$ levels as treatments in comparison with two standard strains in rice (var. ADT43) under pot culture conditions. Among three different moisture regimes, 50\% field capacity with AztRMD2 showed the maximum plant height $(90 \mathrm{~cm})$. With regard to fertilizer $\mathrm{N}$ levels used, AztRMD2 isolate with $75 \%$ recommended dose of $\mathrm{N}$ showed higher plant height $(80 \mathrm{~cm})$. The results indicated that AztRMD2 performed better than the standard strains and other treatments in increasing the growth of rice under water limiting conditions and reduced fertilizer $\mathrm{N}$ application.

\section{Introduction}

Azotobacter is the genus of great interest in agricultural application due to their free nitrogen fixing ability. It is a free living, asymbiotic nitrogen fixer and mostly abundant in plant rhizosphere and phyllosphere region. Azotobacter are the 
unique biofertilizers to maintain the $\mathrm{N}$ level in agricultural soil and synthesize the plant growth promoting hormones as indole acetic acid and gibberellins. The EPS from Azotobacter in its microenvironments facilitates its survivability in diverse agricultural fields. The EPS of Azotobacter are copolyuronans ubiquitous nature of alginates (Elsayed et al., 2013) commercially used in plant tissue culture to produce insoluble artificial seeds, immobilizing enzymes by entrapment, as food and wound dressing substances. Thus the industrial application of alginate is well understood (Hay et al., 2010). Aside from industrial application, Azotobacter alginate plays a key role in encystment, to provide protection against desiccation and predation by protozoa or phage attack (Hynes et al., 2008), or affect the penetration of antimicrobial agents and toxic metals (Aleem et al., 2003). This polysaccharide also protects nitrogenase against high oxygen concentration and also participates in interaction between plants and bacteria (Mandal et al., 2008). Azotobacter EPS in the soil habitat play key roles in ecosystems functioning through controlling nutrient cycling reaction essential for maintaining soil fertility and also contributing to the genesis and maintenance of soil structure under conventional, biotic, and abiotic stressed soil environment. The major issues in production of efficient biofertilizers include the characteristics of high rate of dinitrogen fixation, wide range of antagonistic activity towards phytopathogens, and the ability to produce EPS, siderophores, vitamins, and growth factors in agricultural perspective. Hence, Azotobacter EPS adopt different mechanisms to promote plant growth and other rhizobacteria. These beneficial traits are promising environment friendly tools for sustainable agriculture and its own survivability (Ahemad, 2012).

Salinity is a major environmental constraint to crop productivity throughout the arid and semi-arid regions of the world. A third of arable land resources in the world are affected by salinity (Saghafi et al., 2013). Seed germination is an important factor limiting plant growth and the saline condition can decrease seed germination either by creating osmotic potential that prevents the absorption of water by toxic effects resulting high concentration of $\mathrm{Na}^{+}$in the soil. Thus, the limitation of water absorption can cause various structural, physiological and biochemical modifications of seeds that can reduce the rate of germination and retard plant development. Azotobacter strains, which have the properties like highest nitrogen fixing and better EPS production ability that might flourish well in most of the geographical niches should be considered for next generation inoculants. Hence, understanding and manipulating this feature is of great agroecological interest for future crop husbandry.

EPS production is directly related to alginate production and encystment process. Cyst of Azotobacter is of great interest for liquid biofertilizer production. Liquid biofertilizers have some more advantages over solid biofertilizers as cyst contains special nutrients that ensure longer shelf life, improved survival rate on seeds and soil, drought tolerance, very high enzymatic activity, contamination free and easy handling. A mutant of A.vinelandii deficient in alginate production showed lower encystment ability compared with higher alginate producing strain. The metabolically active cells are able to produce high level of EPS, which may play a protective mechanism by forming a rigid gelatinous structure surrounding the cells called cyst coat. EPS are essential molecules to maintain cellular hydration and biofilm formation under desiccating conditions. The polysaccharides are able to form various structures within a biofilm and may interact with a wide range of other molecular species, including lectins, proteins, lipids, etc., (Mann and Wozniak, 2012). Biofilm is a crucial 
phenotype in the survival of bacterial populations in adverse environmental condition. EPS of Azotobacter plays a major role in immobilization of heavy metals. They directly bind and uptake heavy metals like $\mathrm{Cd}$ and $\mathrm{Cr}$ in the contaminated soils. The metal absorption (like $\mathrm{Cd}^{2+}, \mathrm{Cu}^{2+}, \mathrm{Pb}^{2+}, \mathrm{Zn}^{2+}$ ) behavior of alginate from Azotobacter in soil and water environment helps by removing toxic metals or by creating microenvironment of essential metal ions to maintain soil ecology and accelerate the normal growth of plant (Aisha and Al-Rajhi, 2013).

EPS is more effective in binding or aggregating the soil. It also reduces the bulk density of Greenfield sandy loam and increased hydraulic conductivity in neutral soil. Moreover, the polysaccharide rich soil provides a cementing action to increase stability for soil aggregation (). Soil aggregate stability increases biomass and decomposition of organic materials by PGPRs (Ahemad and Khan, 2012). This increased soil porosity and reduced bulk density allows retention and exchange of air and water. This pore space provides zones of weakness through which plant roots can grow easily. Hence, in the present investigation, attempts have been made to isolate efficient EPS producing and cyst forming Azotobacter from different locations of Tamil Nadu for better performance under salt stress condition of rice ecosystem.

\section{Materials and Methods}

All the laboratory and pot culture experiments were conducted at the Department of Agricultural Microbiology, Tamil Nadu Agricultural University (TNAU), Coimbatore. The standard strain of Azotobacter chroococcum (Ac1) maintained at the Biofertilizer Production and Quality Control Laboratory, TNAU and the standard strain of Azotobacter venilandii (MTCC2460) was obtained from Microbial Type Culture Collection and Gene Bank, India were used in the study.

\section{Isolation and purification of Azotobacter isolates}

The rhizosphere soil samples were collected from different rice growing places such as Paramakudi, Madurai, Ramnad and different region of Kanyakumari and Nilgiri districts. Azotobacter isolates was isolated by following dilution plating method using Waksman No 77 medium. A total of 30 selected isolates were purified on Waksman No 77 medium by streak plate method and designated. Stock cultures were made in Waksman No 77 broth containing 60\% (w/v) glycerol and stored at $-20^{\circ} \mathrm{C}$.

The selected isolates were subjected to a set of morphological and biochemical tests for the purpose of identification. The cellular morphology like shape and cell arrangement, Motility test, Gram staining, colony characters viz., colour, elevation, shape were observed under microscope and pigment production, polysaccharide production in Waksman No 77 media were recorded.

The biochemical tests viz., catalase test, Oxidase, Indole formation test, Starch hydrolysis test, Gelation liquefaction was carried out for identification of genus.

\section{Selection of efficient EPS producing and cyst forming Azotobacter isolates}

Cell dry weight determination and Polysaccharide determination (Jarman et al., 1978) was evaluated. Furtherly, for the selection of efficient Azotobacter isolates, following studies on cyst formation by Azotobacter (Socolofsky and Wyss, 1962) was performed. 
Induction of cyst formation under specific micronutrient limiting conditions by omission of specific single micronutrient such as calcium, iron, magnesium, and molybdenum from the Burk's $\mathrm{N}$ free medium.

Moreover, elimination of combination of two micronutrients on cyst formation in the flasks viz., $\mathrm{F}_{1}$ - without $\mathrm{Ca} \& \mathrm{Fe} ; \mathrm{F}_{2}$ - without $\mathrm{Ca} \&$ $\mathrm{Mg} ; \mathrm{F}_{3}$ - without Fe \& $\mathrm{Mg} ; \mathrm{F}_{4}$ - without Fe \& $\mathrm{Mo} \mathrm{F}_{5}-\mathrm{Ca} \& \mathrm{Mo} ; \mathrm{F}_{6}$ - without $\mathrm{Mg} \&$ Mo was also determined. A set of flasks containing medium from which none of the micronutrient had been omitted again served as Controls.

Induction of cyst formation under reduced concentration of sucrose i.e., three different concentration of sucrose viz., $0.5 \%$ (normal concentration) and two reduced concentrations viz., $0.1 \%$ and $0.05 \%$ were studied.

Besides that induction of cyst formation with n-butanol as carbon source in liquid Burk's medium containing either $\mathrm{n}$ - butanol or $\mathrm{n}-$ butanol agar extract was also performed. The nitrogen fixing capacity of the isolates was evaluated by Acetylene Reduction Assay in the Gas Chromatograph (Chemito GC 7610) following the standard procedure (Burris, 1974).

For knowing the salt tolerance, Waksman No 77 broth containing different concentration of $\mathrm{NaCl}$ viz. $0 \%, 2 \%, 4 \%, 6 \%$ and $10 \%$ were inoculated and incubated at $28{ }^{\circ} \mathrm{C}$ for 48 hours and compared with the Control.

The selected elite Azotobacter isolate AztRMD2 from above experiments was then studied for formation of artificial soil aggregates (Ramasawmy et al., 1992) in comparison with two standard strains AztTNAU and AztMTCC2460.

\section{Pot culture experiment}

The impact of Azotobacter isolate AztRMD2 and the standard strains (AztAc1 and AztMTCC2460) on the growth of rice (var.ADT43) was evaluated. The Azotobacter isolate AztRMD2 and standard strains (AztAc1 and AztMTCC2460) were grown in Waksman No. 77 broth till the population reached to $10^{10}$ cells $\mathrm{ml}^{-1}$. Carrier based inoculum using lignite as carrier material was prepared with the broth culture and used as bacterial inoculants for this experiment. Surface sterilized seeds were treated with the above strains of Azotobacter separately after preparing semisolid slurry by mixing with carboxy methyl cellulose as an adhesive and shade dried for 20-30 min before sowing. Rice seeds were sown directly in the pot. The treatments were replicated thrice in a randomized block design. Two different experiments were carried out under the pot culture conditions.

\section{Experiment 1: Different moisture regimes}

The effect of inoculation of Azotobacter strains at three different moisture regimes 50 $\%$ field capacity $\left(\mathrm{I}_{1}\right)$, alternate wetting and drying $\left(\mathrm{I}_{2}\right)$ and submerged condition $\left(\mathrm{I}_{3}\right)$ was studied under pot culture conditions. The treatment details are : $\mathrm{T}_{1}-$ AztRMD2 $+\mathrm{I}_{1} ; \mathrm{T}_{2}$ AztMTCC2460 + $\mathrm{I}_{1} ; \mathrm{T}_{3}-$ AztTNAU + $\mathrm{I}_{1} ; \mathrm{T}_{4}-$ AztRMD2 + $\mathrm{I}_{2} ; \mathrm{T}_{5}-\mathrm{AztMTCC} 2460+\mathrm{I}_{2} ; \mathrm{T}_{6}-$ AztTNAU $+\mathrm{I}_{2} ; \mathrm{T}_{7}-$ AztRMD2 + $\mathrm{I}_{3} ; \mathrm{T}_{8}-$ AztMTCC $2460+\mathrm{I}_{3}$ and $\mathrm{T}_{9}-$ AztTNAU $+\mathrm{I}_{3}$.

\section{Experiment 2: Different nitrogen levels}

The performance of Azotobacter strains with three different nitrogen levels viz., $50 \%\left(\mathrm{~F}_{50}\right)$, $75 \%\left(\mathrm{~F}_{75}\right)$ and $100 \%\left(\mathrm{~F}_{100}\right)$, were applied for rice and an unfertilized control was also maintained in pot culture conditions. The treatment details are : $\mathrm{T}_{1}-$ AztRMD2 $+\mathrm{F}_{50} ; \mathrm{T}_{2}$ - AztMTCC2460 + $\mathrm{F}_{50} ; \mathrm{T}_{3}-$ AztTNAU + $\mathrm{F}_{50}$; 
$\mathrm{T}_{4}-\mathrm{AztRMD} 2+\mathrm{F}_{75} ; \mathrm{T}_{5}-\operatorname{AztMTCC} 2460+$ $\mathrm{F}_{75} ; \mathrm{T}_{6}-\mathrm{AztTNAU}+\mathrm{F}_{75} ; \mathrm{T}_{7}-\mathrm{AztRMD} 2+$ $\mathrm{F}_{100} ; \mathrm{T}_{8}-$ AztMTCC2460 $+\mathrm{F}_{100} ; \mathrm{T}_{9}-$ AztTNAU $+\mathrm{F}_{100} ; \mathrm{T}_{10}-$ Uninoculated unfertilized control. 100\% recommended dose of $\mathrm{P}$ and $\mathrm{K}$ were applied basally in all the treatments.

The following observations on plant growth parameters viz., shoot length, root length and plant dry weight were recorded 30, 60 and 90 days after sowing (DAS).The data generated from the experiment were subjected to statistical analysis as suggested by Panse and Sukhatme (1976) using AGRESS software package.

\section{Results and Discussion}

In the present investigation, isolation, morphological and biochemical characteristics of the all the isolates were carried out for confirmation at genus level as Azotobacter. The isolates were screened for the production of EPS and formation of cyst in the nutrients limiting conditions. The production of EPS in different salt concentration was carried out to obtain the efficient strains of salt tolerant Azotobacter isolates. The efficient Azotobacter isolates were screened under in vitro conditions on rice for the growth parameters by pot culture experiments.

After isolation and purification, all the 30 isolates were subjected to preliminary identification. Among them based on morphological and biochemical characterization, 10 efficient Azotobacter isolates viz., AztPMK1, AztPu1, AztPMK2, AztPa, AztMDU1, AztKa, AztMDU2, AztMDUT1, AztRMD1, AztRMD2 were selected and the production of EPS was compared with the standard cultures, AztAc1 (Azotobacter chroocochum) and AztMTCC 2460 (Azotobacter venilandii). The isolate AztRMD2 recorded maximum polysaccharide production $(1.755 \mathrm{~g} / 50 \mathrm{ml})$ in Waksman No 77 broth. Preliminary experiments were performed in order to determine the incubation time for optimum recovery of EPS in Azotobacter cultures. The result was in accordance with the Lozano et al., (2011). Thus, culture media samples were removed at intervals and quantitative extractions of EPS were made. According to these results maximum EPS recovery could be obtained in 5-day-old cultures (Table 1). Hence the incubation period was standardized as five days for EPS extraction and quantification in further experiments. The yield of EPS production is negatively correlated with biomass production and positively correlated with carbon source consumption. The yield of EPS increases when the biomass production decreases. And also the specific yields of EPS production were higher as the initial concentration of substrates increased (Hay et al., 2010). From these studies, three Azotobacter strains viz., AztRMD2, AztPMK1 and AztPMK2 were selected for further experiments.

Segura (2003) found that cyst formation can be induced in Azotobacter by addition of $0.3 \%$ n-butanol as the carbon source to the medium on which the organisms are grown. However, they were unable to induce cyst formation in liquid Burk's medium with sucrose as the carbon source. Thus, the view that cyst formation in Azotobacter does not occur in media containing sucrose as the carbon source has gained widespread acceptance in recent years. The present study however shows this view to be untenable, since cyst formation was induced in liquid Burk's medium, with sucrose as the carbon source, by elimination of one or more of the micronutrients normally present in the medium. In the recent study, elimination of combination of two micronutrients from the medium was found to bring about much greater increase in the number of cysts formed 
than elimination of a specific micronutrient. Thus, the effects of micronutrients deficiencies in inducing cyst formation seem to be additive. Another interesting observation is that the number of cysts formed in response to elimination of combination of two micronutrients is very nearly the same, no matter what two micronutrients are eliminated. This may indicate that one or more of the micronutrients are able to substitute for one another.

During the course of this study, it was also shown that cyst formation can be induced by reduction of the sucrose content of the medium in which the organisms are grown and, as was already shown (Gauri et al., 2011), incorporation of $0.3 \%$ n-butanol as the carbon source into the solidified Burk's medium on which the organisms are grown also induces cyst formation. Thus, it would seem that, the Azotobacter cyst may represent a survival state formed in response to a variety of detrimental changes in the environment. It would further seem that induction of cyst formation in response to mineral deficiencies, as was accomplished in the present study, would come closer to representing the mechanisms controlling cyst formation in nature than would cyst formation in response to the presence of such compounds as butanol in the environment.

Salt stress affects both bacteria and plants in two ways: it induces ionic stress due to the high concentration of ions and also osmotic stress through the change in the solute concentration around the cells, producing water deficit and desiccation. Azotobacter subjected to salt stress may undergo morphological alterations, leading to changes in cell morphology and size or modifications in the pattern of EPS and lipopolysaccharides (LPS) (Vanderlinde et al., 2010). In the present study, exopolysaccharide production was determined in different salt concentration. Some strains of Azotobacter are able to grow at $\mathrm{NaCl}$ concentrations as high as $4 \%$ and others cannot grow, when $\mathrm{NaCl}$ concentration is above $6 \%$. The result was in accordance with the Kohler and Caravaca, (2010). During the course of this study it was also shown that the polysaccharide production was minimum in the $4 \%$ salt concentration. It is mainly due to the loss of intracellular water, which imposes a water deficit because of osmotic effects on a wide variety of metabolic activities (Fatnassi et al., 2011) and the maximum polysaccharide production was noticed in the medium containing $\mathrm{NaCl}$ in the normal concentration. These results are in conformity with the findings of Alavi et al., (2013).

Soil structure has a strong impact on a range of processes influencing crop yield. The basic units of soil structure, named aggregates, comprise solid material and pores. These aggregates determine the mechanical and physical properties of soil such as retention and movement of water, aeration, and temperature (Kohler and Caravaca, 2010). Plant roots contribute to soil organic material, and thereby to soil aggregate stability, directly through the root material itself and indirectly through stimulation of microbial activity in the rhizosphere. It is generally believed that microbial action on soil aggregation is due to the production of EPS. In the present investigation Azotobacter isolates were examined for improving aggregation of rice field soil. The results indicated a great influence of Azotobacter on soil physical conditions at varying degree of aggregation treatments. The improvement in the structure of treated soils was assessed by the measurements of soil porosity and bulk density. Among the isolates, AzRMD2 performed well recording higher pore space in soil. The pore space had increased in all the treatments, when compared to Control. In soil, pore space increased to $62.5 \%$ by 
inoculating AzRMD2 isolate over the Control. The pore space may be increased due to the aggregation by the influence of microorganisms. In a similar study, a characteristic improvement of macro pores (porosity, pore size distribution, pore continuity, stability of the pore system, resiliency), infiltration, drainage and aeration by inoculation of bacteria was observed by Freitas et al., (2011).

Table.1 Cell dry weight and Exopolysaccharide production by different Azotobacter isolates

\begin{tabular}{|c|c|c|}
\hline Azotobacter Isolates & $\begin{array}{l}\text { Cell dry weight }(\mathrm{g} / 50 \mathrm{ml}) \\
\text { after } 5 \text { days growth }\end{array}$ & $\begin{array}{c}\text { Exopolysaccharide content } \\
(\mathrm{g} / 50 \mathrm{ml})\end{array}$ \\
\hline AztPMK1 & 0.244 & 0.783 \\
\hline AztPu1 & 0.186 & 0.450 \\
\hline AztPMK 2 & 0.283 & 1.353 \\
\hline AztPa1 & 0.543 & 0.251 \\
\hline AztMDU1 & 6.021 & 0.550 \\
\hline AztKa & 19.416 & 0.030 \\
\hline AztMDU2 & 1.599 & 0.332 \\
\hline AztMDUT1 & 2.086 & 0.492 \\
\hline AztRMD1 & 0.019 & 1.560 \\
\hline AztRMD 2 & 0.100 & 1.755 \\
\hline AztTNAU* & 2.279 & 0.830 \\
\hline AztMTCC 2460* & 0.162 & 1.570 \\
\hline
\end{tabular}

Fig.1 Effect of Azotobacter inoculation at different moisture regime on Plant height (var ADT 43) under pot culture conditions

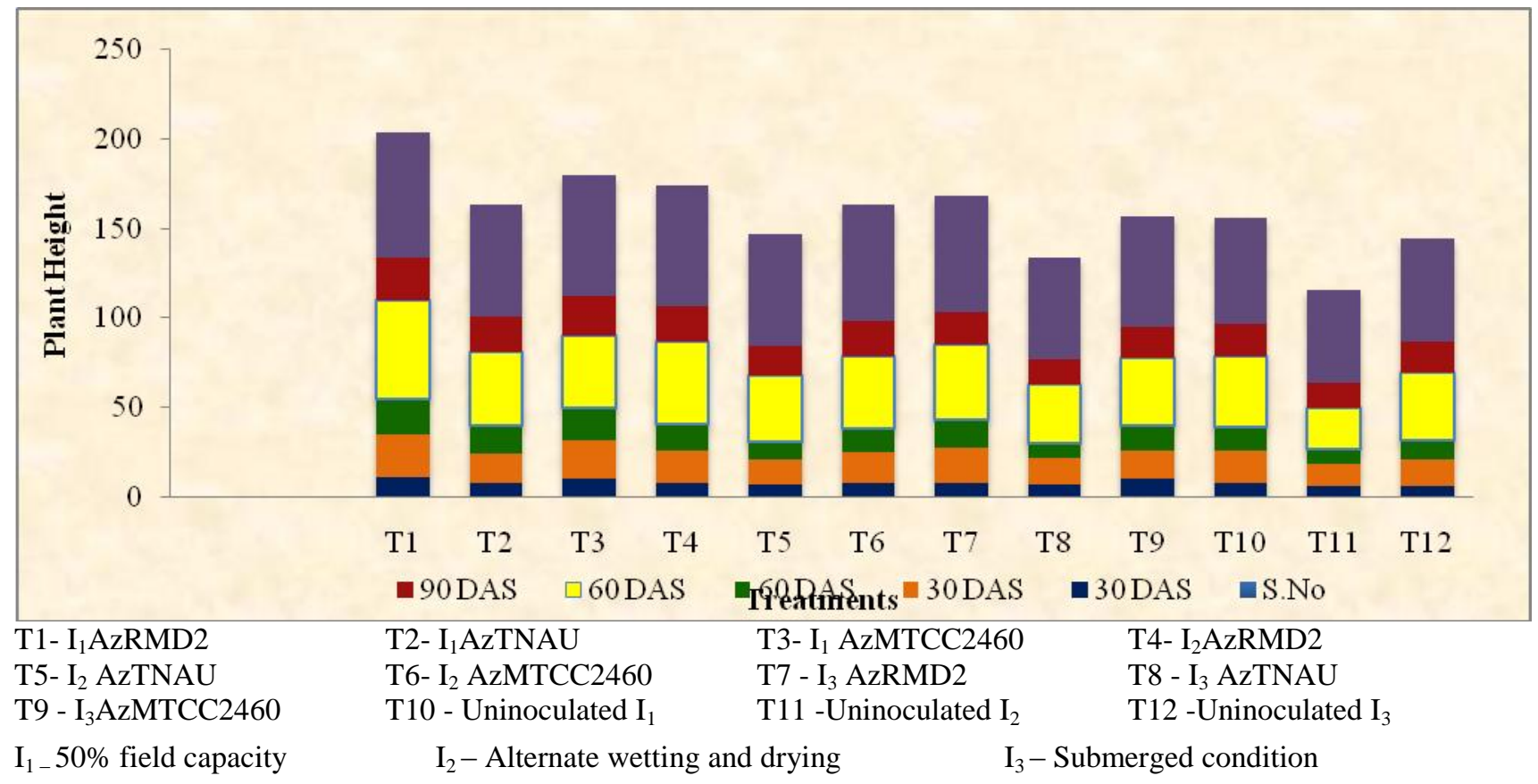


Plate.1 Effect of inoculation of selected and standard strains of Azotobacter isolates on growth of rice var. (ADT 43) under pot culture condition

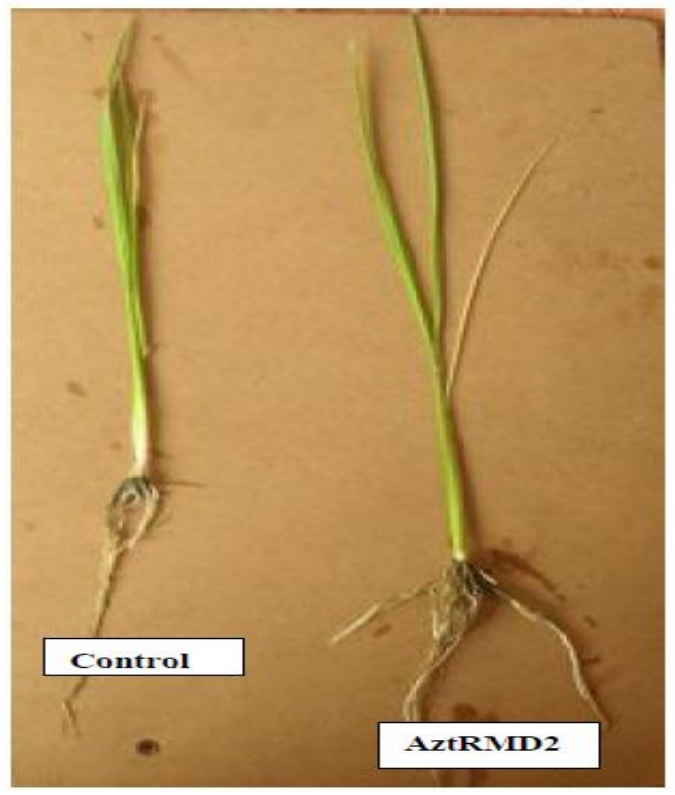

a.

Effect of Azotobacter isolates on different moisture regime. (50\% field capacity)

The bulk density was reduced to $0.5 \mathrm{~g} / \mathrm{cc}$ with the inoculation of AzRMD2 isolate. Reduction of bulk density may be due to the addition of materials that would automatically decrease bulk density, because the added material is of lower density. Further, the development of good aggregate and structure by the binding substances produced by the added bacterium might have reduced the volume of soil solids and consequently the bulk density. These results confirm the findings of Maqubela et al., (2009), who observed a decrease in bulk density of soil on the addition of organic matter. This reduction might occur directly by dilution of the soil matrix with a less dense material or indirectly by the improvement of aggregate stability. In the present study the aggregate stability was higher (45 drops) in the soil, with the inoculation of AztRMD2 isolate. This is confirmation with the result of Tikhonovich and Provorvo (2011), who found that addition of organic matter improved the aggregation stability. The EPS from Azotobacter augment

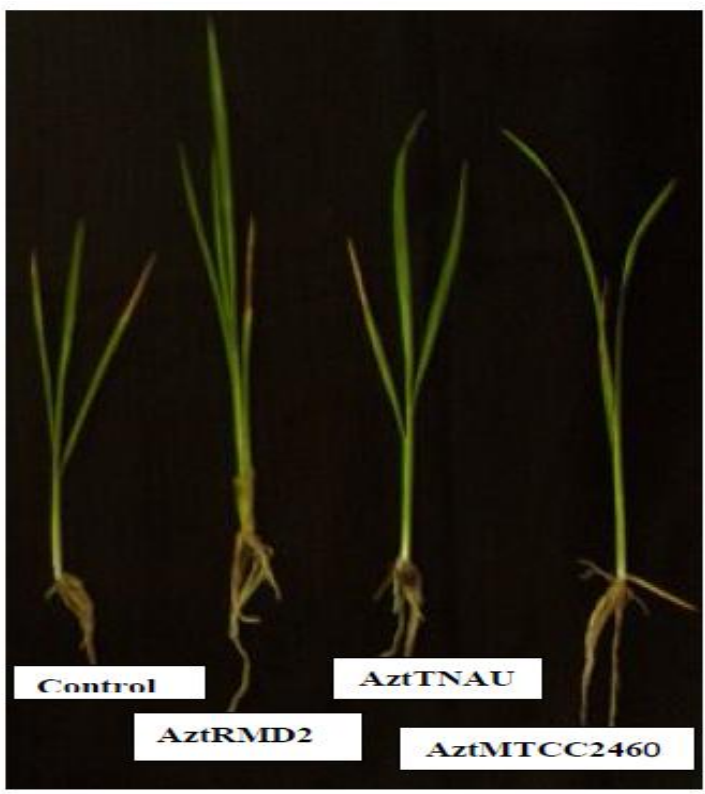

b. Effect of Azotobacter isolates on different nitrogen recommendation. (75\% nitrogen)

the aggregate stability. It is imperative that the artificially formed aggregates had more stability and will throw more information, if tested for crop production and soil characteristics than applied in soil.

Interestingly, the isolate AztRDM2 recorded higher population in $50 \%$ field capacity. This survival ability under these conditions may be due to the EPS production. The EPS layer may maintain a hydrated microenvironment around microorganisms during desiccation. Gordon et al., (2010) demonstrated that desiccation survival of Rhizobium sp. and Pseudomonas sp., respectively required an increased EPS production.

As expected, the increase in the population of EPS-producing strain AzRDM2 in the rice rhizosphere after inoculation, significantly increased the shoot length and root length ratio, irrespective of the water conditions. Similar results were obtained for wheat plants inoculated with either Azotobacter or 
Azospirillum (Ashraf et al., 2006). This significant increase in root adhering soil mass around the roots of rice plants inoculated with Azotobacter isolate AzRDM2 could be the result of either an increase in soil adhesion to roots or a higher soil aggregate stability around roots, or both. This aggregation effect of Azotobacter strain AzRDM2 may be due to the EPS production. Purified xanthan and alginate (produced by Xanthomonas sp. and Azotobacter vinelandii, respectively) can improve aggregate formation. The polysaccharides are apparently adsorbed on soil particle surfaces and cement particles together (Diaz-Barrera et al., 2009)

On the other hand, it was shown previously that microbial biomass and polysaccharide production are increased in association with the stimulation of microbial populations in the rhizosphere of various plants. The factors that favor root cap polysaccharide production may be expected to improve soil adhesion to roots or root adhering soil aggregation. Hence, the effect of Azotobacter strain AzRDM2 in rice rhizosphere on soil aggregation may also be partly indirect, through a stimulation of root exudation. On plantlets subjected to water stress, whatever the inoculation treatment, the root adhering soil or root tissue ratio values were lower than those of plantlets growing under normal water supply conditions. Of particular interest in this study is the finding that this root adhering soil of stressed and inoculated plants is as great as that of noninoculated non stressed plants. This suggests that inoculation of rice with Azotobacter strain AzRDM2 may limit the negative effect of dry conditions on soil aggregation. This effect may also be related to the production of EPS by Azotobacter isolate AzRDM2. Microbial EPS may both increase water holding capacity of soil and reduce water loss during desiccation (Tank and Saraf, 2010).

Rice productivity is severly restricted due to available $\mathrm{N}$ and the conventional biological nitrogen fixation has only a limited potential to render rice independent of external source of N. It is essential to develop and strengthen some innovative approaches to harness biological nitrogen fixation (Bhattacharyya and Jha, 2012). These approaches should aim at developing a more efficient association between rice and diazotrophs by creating relationship between selected diazotrophs and rice. The experiment on non-leguminous crops with diazotrophic bacteria has been studied for many years with the expectation that these bacteria would fix dinitrogen gas and provide combined nitrogen to the plant for enhanced crop production (Saadatnia and Riahi, 2009).

In the present study, the population of Azotobacter and biometric observations were recorded in the pot culture experiment (plate 1). The maximum population was recorded by Azotobacter isolate on 30 DAS in treatment 75\% recommended dose $\mathrm{N}+$ AzRMD2 (26 $\left.\mathrm{x} 10^{3} \mathrm{cfu} \mathrm{g}^{-1}\right)$. The results showed that at 90 DAS, the biometric observations such as plant height were increased significantly with the inoculation of $75 \%$ recommended dose $\mathrm{N}+$ AzRMD2 with $80 \mathrm{~cm}$ (Fig. 1), may be due to continuous supply of nutrients throughout its growth stage from Azotobacter. Leaching loss of nutrients must have been minimized by use of Azotobacter, which has an ability to mobilize nutritionally important elements from non-usable forms to usable forms. According to Sahoo et al., (2013), in addition to its high $\mathrm{N}$ fixation, Azotobacter is known to synthesize growth substances such as IAA and other auxins and vitamin $B$, which might have also helped in increasing the plant height.

Based on the presence of desirable characters such as EPS production, cyst formation, salinity tolerance and soil aggregation, the Azotobacter isolate AztRMD2 was selected for studying its performance in comparison with two standard strains in rice (var. ADT43) 
under pot culture conditions. Azotobacter strains were inoculated a different moisture regimes and fertilizer $\mathrm{N}$ levels as treatments. Among the three different moisture regimes, $50 \%$ field capacity with AztRMD2 showed the maximum plant height $(90 \mathrm{~cm})$. With regard to fertilizer $\mathrm{N}$ levels used, AztRMD2 isolate with $75 \%$ recommended dose of $\mathrm{N}$ showed higher plant height $(80 \mathrm{~cm})$. The results indicated that AztRMD2 performed better than the standard strains and other treatments in increasing the growth of rice under water limiting conditions and reduced fertilizer $\mathrm{N}$ application.

\section{References}

Ahemad, M. 2012. Implications of bacterial resistance against heavy metals in bioremediation: a review. IIOABJ, 3: 3946.

Ahemad, M. and M.S. Khan. 2012. Evaluation of plant growth promoting activities of rhizobacterium Pseudomonas putida under herbicide-stress. Ann. Microbiol., 62: $1531-1540$

Aisha, M.H. and Al-Rajhi. 2013. Impact of biofertilizer Trichoderma harzianum Rifai and the biomarker changes in Eruca sativa L. plant grown in metal-polluted soils. World. Appl. Sci. J., 22: 171-180.

Alavi, P., M.R. Starcher, C. Zachow, H. Müller and G. Berg. 2013. Root-microbe systems: the effect and mode of interaction of stress protecting agent (SPA) Stenotrophomonas rhizophila DSM14405. Front. Plant Sci., 4:141.

Aleem, A., J. Isar and A. Malik.2003. Impact of long-term application of industrial wastewater on the emergence of resistance traits in Azotobacter chroococcum isolated from rhizospheric soil. Bioresour. Technol., 86:7-13.

Ashraf,M.., S. Hasnain and O. Berge. 2006. Effect of exo- Polysaccharides producing bacterial inoculation on growth of roots of wheat (Triticum aestivum L.) plants grown in a salt-affected soil. Int. J. Environ. Sci.
Tech., 3 (1): 43-51.

Bhattacharyya, P.N. and D.K. Jha. 2012. Plant growth-promoting rhizobacteria (PGPR): emergence in agriculture. World $\mathrm{J}$. Microbiol. Biotechnol, 28: 1327-1350.

Burris, R.H. 1974. Biochemical genetics of nitrogenase. In: Biology of Nitrogen Fixation. (Ed.) A. Quispel. North Holland Publishing Co., pp. 3-42.

Diaz-Barrera, A., P.Silva, R.Avalos and F. Acevedo. 2009. Alginate molecular mass produced by Azotobacter vinelandii in response to changes of the $\mathrm{O}_{2}$ transfer rate in chemostat cultures. Biotechnol. Lett, 31:825-829.

Elsayed, N.S., M. Aboulwafa, K. Aboshanab and N. Hassouna. 2013. PHB production in Azomonas, Acinteobacter and Bacillus species: isolation, screening and identification. Arch. Clin. Microbiol, in press.

Fatnassi, I.C, S.H.Jebara and M. Jebara. 2011. Selection of symbiotically efficient and high salt-tolerant rhizobia strains by gamma irradiation. Ann Microbiol., 61: 291- 297.

Freitas, F., V.D. Alves and M.A.M. Reis. 2011. Advances in bacterial exopolysaccharides: from production to biotechnological applications. Trends Biotechnol, 29:388398.

Gauri, S.S., S. Archanaa, K.C. Mondal, B.R. Pati, S.M. Mandal and S. Dey. 2011. Removal of arsenic from aqueous solution using pottery granules coated with cyst of Azotobacter and Portland cement: characterization, kinetics and modeling. Bioresour. Technol., 102:6308-6312.

Ghafoor A. and B.H.A. Rehm. 2010. Bacterial biosynthesis of alginates. J. Chem. Technol. Biotechnol., 85:752-759.

Hay, I.D., Z.U. Rehman, A. Ghafoor and B.H.A. Rehm. 2010. Bacterial biosynthesis of alginates. J. Chem. Technol. Biotechnol., 85:752-759.

Hynes, R.K., G.C.Leung, D.L. Hirkala and L.M. Nelson.2008. Isolation, selection, and characterization of beneficial rhizobacteria from pea, lentil and chickpea grown in 
Western Canada. Can. J. Microbiol., 54: 248-258.

Jarman, T.R., L. Deavin, S. Slocombe, and R.C. Righelato. 1978. Investigations of the effect of environmental conditions on the rate of exopolysaccharide synthesis in Azotobacter vinelandii. J. Gen Microbiol, 107: 59-64.

Kohler. J. and F. Caravaca. 2010. An AM fungus and a PGPR intensify the adverse effects of salinity on the stability of rhizosphere soil aggregates of Lactuca sativa Roldan. Soil Biol. Biochem., 42:429-434.

Lozano, E., E. Galindo and C.F. Pena. 2011. Oxygen transfer rate during the production of alginate by Azotobacter vinelandii under oxygen limited and non-oxygen-limited conditions. Microb. Cell Factories, 10:13.

Mandal, S.M., B.R. Pati, A.K.Das and A.K. Ghosh. 2008. Characterization of a symbiotically effective Rhizobium resistant to arsenic: Isolated from the root nodules of Vigna mungo (L.) Hepper grown in an arsenic-contaminated field. J. Gen. Appl. Microbiol., 54:93-99.

Mann, E.E. and D.J. Wozniak. 2012. Pseudomonas biofilm matrix composition and niche biology. FEMS. Microbiol. Rev.J, 1574.

Maqubela, M.P., P.N.S. MnkenI, O. Malam issa, M.T.Pardo, and L.P.Dacqui. 2009. Nostoc cyanobacterial inoculation in South African agricultural soils enhances soil structure, fertility, and maize growth. Plant Soil, 315:79-92.

Ramasawmy, K., V. Gomathy and R. Subramanian.1992. Isolation and characterization of microbial products involved in stable soil aggregation. In: Final report submitted to ICAR, Adhoc Research Project Dept. of environmental sciences, Tamil Nadu Agrl. University, Coimbatore, India.p 115.

Saadatnia, H. and H. Riahi. 2009. Cyanobacteria from paddy fields in Iran as a biofertilizer in rice plants. Plant Soil Environ, 55, (5): 207.212.

Saghafi, K., J. Ahmadi, A. Asgharzadeh and S. Bakhtiari. 2013. The effect of microbial inoculants on physiological responses of two wheat cultivars under salt stress. Int. J. Adv. Biol. Biom. Res., 1:421-431.

Segura, D., J. Guzmaan and G. Espin.2003. Azotobacter vinelandii mutants that overproduce poly-beta-hydroxybutyrate or alginate. Appl. Microbiol. Biotechnol, 63:159-163

Socolofsky, M. D. and O. Wyss. 1962. Resistance of Azotobacter cyst. J. Bacteriol., 84:119-124.

Tank, N. and M. Saraf. 2010. Salinity-resistant plant growth promoting rhizobacteria ameliorates sodium chloride stress on tomato plants. J. Plant Interact, 5: 51-58.

Tikhonovich, I.A. and N.A. Provorvo. 2011. Microbiology is the basis of sustainable agriculture: an opinion. Ann. Appl. Biol., 159: 155-168.

Vanderlinde, E.M., J.J. Harrison, A. Muszynski, R.W. Carlson, R.J. Turner and C.K. Yost. 2010. Identification of a novel ABCtransporter required for desiccation tolerance and biofilm formation in Rhizobium leguminosarum bv. viciae 3841. FEMS Microbiol. Ecol., 71: 327-340.

\section{How to cite this article:}

Sivapriya, S.L. and Pasupuleti Reddy Priya. 2017. Selection of Hyper Exopolysaccharide Producing and Cyst Forming Azotobacter Isolates for Better Survival under Stress Conditions. Int.J.Curr.Microbiol.App.Sci. 6(6): 2310-2320. doi: https://doi.org/10.20546/ijcmas.2017.606.274 